\title{
Encuesta de actitudes sobre el Aborto y su Legislación.
}

\section{Tomada entre Médicos de Bogotá}

Como parteconstitutiva del relato sobre ABORTO, y con el ánimo de contribuir a esclarecer en forma parcial uno de los aspectos tocantes al mismo, la Sociedad Colombiana de Obstetricia y Ginecología creyó oportuno explorar las opiniones y actitudes que sobre el aborto y su legislación sustentan los Médicos que ejercen su profesión en Bogotá.

Aunque el ideal hubiese sido recciger la opinión de todo el cuerpo médico colombiano, el tiempo y los recursos solamente permitieron esta primera aproximación.

Con el desarrollo de esta encuesta., básicamente se ha buscado conocer:

a. La opinión del cue po médico respecto a la necesidad o no de modificar la legislación vigente sobrr aborto, y en caso de modificarse en qui sentido se expresaría esa necesidad: hacia una mayor restricción o hacia una apertura de la legislación.

b. El nivel de conocimientos que sobre el tema pudieran tener los encuestados, y

c. La actitud de los médicos ante problemas y situaciones que competen directamente con el ejercicio profesional.

Es evidente que frente a una tendencia cada vez más generalizada a la liberalización de las legislaciones en relación con el aborto en todo el mundo contemporáneo, es claramente pertinente comenzar a conocer qué opina el cuerpo médico de Clolombia sobre el problema, su dimensión y características, sobre la legislación vigente y explorar también cual sería el grado de participación profesional, si se modificara la legislación. Finalmente, en ausencia de estudios previos similares y frente al proyecto de ley actualmente en discusión en el parlamento, el estudio aquí citado cobra una particular relevancia.
El desarrollo de esta parte del relato se realizó en forma multidisciplinaria con la participación de:

a. Los miembros del comité nombrado por las directivas de la Sociedad Colombiana de Obstetricia y Ginecología para la elaboración del relato sobre aborto: Doctores Martha Laignelet de Medina, Armando Lozano, Guillermo López, Luis Maldonado, Jorge Medina, Germán Montoya, Alvaro Quiroga, Jaime Ruiz, Jorge Villarreal, bajo la coordinación del doctor Jorge Medina.

b. La asesoría técnica de las sociólogas María Cristina de Herrán y Amparo López, y

c. La doctora Patricia Escobar y el doctor Jorge Acevedo del Instituto SER de Investigación.

\section{METODOLOGIA}

Atendiendo a los objetivos de la investigación y al carácter anónimo que deberían tener los médicos respondientes, se optó por el modelo de "encuesta por correo" considerando que con ello aumentaba la posibilidad de obtener una mejor calidad en las respuestas dado que no existiría ningún tipo de presión sobre el médico encuestado a quien se proporcionarían todas las facilidades para que a vuelta de correo, pudiese enviar el formulario diligenciado.

Se diseñó un cuestionario de respuestas cerradas en su mayoría, para evitar la dispersión de la información.

Este instrumento se elaboró respondiendo a las inquietudes planteadas por la Sociedad Colombiana de Obstetricia y Ginecología, y pretendía, básicamente, escoger las opiniones que en relación con el aborto presentan los profesionales médicos residentes en Bogotá, e investigar (aunque no 
exhaustivamente) acerca de los conocimientos que poseen y las actitudes que podrían asumir ellos ante el aborto, según las diferentes circunstancias hipotéticas que se plantean en el cuestionario.

La decisión de tramitar la encuesta por correo planteó la necesidad de utilizar un listado lo más completo posible de los médicos que ejercen su profesión en Bogotá. Luego de varias consultas y en vista de lo incompleto y defectuoso de las fuentes de información oficiales, el comité decidió utilizar un directorio actualizado, gentilmente grupo particularmente importante por su conocimiento del problema, se obtuvo una lista de miembros de la Asociación Colombiana de Médicos de Salud Pública y de allí se extractaron los nombres de los que trabajan en Bogotá, quienes fueron añadidos al directorio de la casa Schering.

El universo resultante de médicos en Bogotá agrupa a un total de 2.557 médicos, 95 de los cuales provienen de la lista de médicos de Salud Pública. La distribución de los médicos resultantes, por especialidad, es la siguiente:

\begin{tabular}{|lcr|}
\hline Especialidad & Número & \%o \\
Medicina General & & 33.0 \\
Ginecología y Obstetricia & 844 & 11.4 \\
Pediatría & 291 & 10.6 \\
Medicina Interna y Psiquiatría & 272 & 18.5 \\
Cirugías & 472 & 20.4 \\
Salud Pública & 522 & 3.7 \\
Otras & 95 & 2.4 \\
Total & 61 & 100.0 \\
\hline
\end{tabular}

ofrecido por los laboratorios Schering Colombiana S. A., cuya cobertura del cuerpo médico en ejercicio, se considera la más completa de la ciudad capital.

A pesar de las consideraciones precedentes, el comité encargado de la coordinación de este relato es plenamente conciente de que el listado utilizado tiene dos posibles fallas; así: a- La desactualizàción ocasionada por la gran movilidad de los médicos dentro de la ciudad, donde se calcula que cincuenta profesionales en promedio cambian de dirección mensualmente. $b-$ Los médicos que por diversos motivos no tienen consultorio y, por lo tanto, no estarían incluídos en el directorio de una organización encargada de informar sobre sus productos. En este grupo podrían encontrarse los médicos de Salud Pública y todos aquellos que trabajan exclusivamente en hospitales docentes o asistenciales y en instituciones de seguridad social.

Con miras a subsanar la falta de participación de los especialistas en Salud Pública,
El procedimiento utilizado es susceptible de diversos sesgos. Así, podría suceder que muchos de los formularios no respondidos correspondan a médicos con un volumen de trabajo superior al promedio; o que una parte importante de las respuestas corresponda a médicos fuertemente a favor o en contra del aborto (interesados en responder) en detrimento de los otros médicos no tan ligados al debate; o que el interés sobre el tema varíe de acuerdo con la especialidad del médico, y en tal forma varíe también la proporción de quienes respondieron; etc.

Lo anterior quiere decir que, al no tener una garantía suficiente de aleatoriedad en las respuestas, no es posible determinar su representatividad, y por lo tanto no es posible expandir la muestra para inferir los resultados estadísticamente atribuíbles a la posición de los médicos de Bogotá.

Por tal motivo los resultados que aquí se presentan y discuten corresponden a las opiniones y actitudes de 648 médicos de 
Bogotá que respondieron a la encuesta. No puede asegurarse que la posición de la totalidad de los médicos de Bogotá sea necesariamente similar.

Aunque lo anterior implica que la encuesta no puede considerarse en rigor: como representativa de los médicos de Bogotá, constituye sin embargo, una medición de las opiniones y actitudes de un número considerable de ellos y como tal es un primer esfuerzo importante y valioso por conocer la posición de este grupo de profesionales con respecto a un tema de gran actualidad.

Una vez definido el universo del estudio, se envió a cada médico una breve carta introductoria acompañada del cuestionario 'diseñado por el comité coordinador del relato, que consta de 10 preguntas acerca de las características personales del médico, de la estructura de su familia y de su actividad profesional; 4 preguntas de opiniones sobre la magnitud y jerarquía del problema y, finalmente, 6 preguntas que consultaban sus opiniones y actitudes con respecto al aborto y a su disposición a efectuarlo si éste llegare a ser permitido bajo alguna de las circunstancias mencionadas en las preguntas anteriores.

Al cuestionario se adjuntó además, una copia de las disposiciones vigentes sobre el aborto en la legislación colombiana, y un sobre estampillado dirigido a la Sociedad Colombiana de Obstetricia y Ginecología con miras a facilitar en lo posible la participación en la encuesta.
Llegada la fecha de corte establecida por el comité coordinador como límite adecuado para el cierre del estudio, sólo 371 médicos habían respondido. Esta situación obligó al grupo de trabajo a replantear su procedimiento y decidió entonces llevar a cabo una segunda ronda utilizando una carta de presentación mucho maś informativa, la cual destacaba la importancia de la participación de todo el cuerpo médico, independientemente de su pespecialidad, en la realización de este estudio. La carta fue muy clara en solicitar a quienes ya habían diligenciado la encuesta en la primera vuelta que ignorasen la segunda.

Este, segundo intento que logró 277 respuestas adicionales llevó el total a 648 cuestionarios para una tasa de respuestas del $25.3 \%$, lo cual se considera cercano a lo que usualmente se obtiene en esta clase de estudios y por tanto aceptable.

Los 648 cuestionarios recibidos hasta el 12 de octubre del presente año representan el matarial de base de este estudio, cuyas etapas de codificación, perforación, análisis de inconsistencia de las encuestas, procesamiento y redacción de un pequeño informe, estuvieron a cargo del Instituto SER de Investigación.

A continuación se presentan las características de la muestra y finalmente los resultados de la encuesta:

CUADRO No. 1

RESPONDIERON A LA ENCUESTA: 648

Especialidad

do Respuestas

olo sobre total de Médicos en Bogotá

Cirugías

Medicina Interna y Psiquiatría

24.1

29.9

Ginecología y Obstetricia

20.8

28.4

17.3

38.5

Medicina General

15.0

11.5

Pediatría

11.6

27.6

Salud Pública

2.5

Más de una especialidad

Otras

5.7

2.9

16.8

31.1 
Si bien es cierto que en razón de su número los especialistas en Medicina Interna y Cirugía aportan el mayor porcentaje de respuestas, fueron como era de esperarse, las Obstetras y Ginecólogos de la capital, quienes en una proporción más elevada dentro de cada especialidad atendieron la solicitud de la Sociedad.

Vale la pena destacar, sin embargo, la relativa apatía de los médicos para pronunciarse frente a un tema de tan vital importancia.

Quienes respondieron a la encuesta fueron en su gran mayoría, médicos del sexo masculino entre 41 y 50 años de edad, casados, católicos, dedicados al ejercicio privado e institucional. La mayoría tienen hijos de ambos sexos, llevan entre 16 y 30 años de ejercicio profesional, se dedican exclusivamente a la prestación de servicios y atienden prioritariamente clientela media y media-baja; por lo tanto, dentro de las limitaciones ya enunciadas del presente estudio, pueden considerarse representativos de la población médica de Bogotá.

\section{RESULTADOS DE LA ENCUESTA}

Los resultados obtenidos pueden clasificarse en dos grupos. El primero presenta las opiniones y el segundo presenta las actitudes de los encuestados frente al problema. La presentación de los resultados seguirá esta clasificación.

La mayoría de los médicos esta de acuerdo en que el aborto constituye un problema de salud que se puede prevenir

CUADRO No. 2

\section{ALGUNAS CARACTERISTICAS DE LOS ENCUESTADOS}

\begin{tabular}{lclr} 
& o & & $\%$ \\
Hombres & 95 & Tienen hijos de ambos sexos & 64 \\
Casados & 87 & $\begin{array}{l}\text { 16 a } 30 \text { años de experiencia } \\
\text { profesional }\end{array}$ & 53 \\
Católicos & 71 & $\begin{array}{l}\text { Dedicados exclusivamente a } \\
\text { la prestación de servicios } \\
\text { En ejercicio privado e }\end{array}$ & $\begin{array}{l}\text { Atiende clientela media y } \\
\text { media baja }\end{array}$ \\
institucional & 42 & 35 & 34 \\
\hline
\end{tabular}

CUADRO No. 3

OPINIONES GENERALES SOBRE EL ABORTO

Respuesta Afirmativa

Mayoritaria

El aborto es un problema de salud

87.5

El aborto constituye un grave problema que puede prevenirse con programas masivos de educación y servicios de planificación familiar

La práctica legalizada del aborto contraría las creencias religiosas y otros valores culturales del país. 


\section{CUADRO No. 4}

\section{EL ABORTO COMO PROBLEMA}

\section{$\%$ de \\ Respuesta Afirmativa}

De Salud Pública

33.0

Etica

26.2

De Salud Individual

17.9

Jurídico

3.2

Varios

13.7

No informaron o más de una respuesta

\section{CUADRO No. 5 \\ OPINIONES SOBRE LA LEGISLACION VIGENTE}

Debe modificarse haciéndola más restrictiva

Debe mantenerse con las restricciones vigen-

tes pero desarrollando los mecanismos para

su aplicación estricta.

Debe mantenerse con las restricciones vigentes

pero sin ninguna modificación adicional

\section{CUADRO No. 6}

\section{OPINIONES SOBRE LA LEGISLACION VIGENTE}

Debe modificarse permitiendo el aborto en algunos casos y bajo ciertas cndiciones por razones médico-terapéuticas (Indicaciones Médicas)

Debe modificarse permitiendo el aborto siempre que el médico y la paciente lo consideren necesario para su salud física, mental y socio-familiar (Indicación Social)

Más de una de las anteriores opiniones 
mediante la educación sexual y los servicios de planificación familiar. Aproximadamente la mitad conceptúa que la legalización contraría creencias religiosas y valores culturales del país, generando al mismo tiempo una demanda adicional de servicios de salud que el estado no estaría en capacidad de atender.

\section{CUADRO No. 4}

Como puede observarse, $33 \%$ de los encuestados, calificaron el aborto como un problema de Salud Pública y $26 \%$ como un problema de carácter etico. Solo un 3.2 \% lo consideró como problema de órden jurídico.

\section{CUADRO No. 5 CUADRO No. 6}

De acuerdo con lo mencionado al exponer la metodología de este estudio y con el ánimo de proporcionar al encuestado la más completa información sobre la legislación vigente, el cuestionario iba acompañado por el texto completo de los artículos que sobre el aborto contempla nuestro Código Penal. Tal como puede apreciarse, un $74.5 \%$ o sea 3 de cada 4 médicos encuestados opinan que la legislación debe modificarse para permitir el aborto bajo ciertas circunstancias o condiciones.. No se encontraron para esta pregunta diferencias significativas entre los dos sexos, pues, $74.5 \%$ de los hombres y $77.4 \%$ de las mujeres opinan que la legislación debe modificarse para permitir el aborto bajo ciertas condiciones.

Al cruzar la opinión sobre la legislación vigente con la especialidad del encuestado:
Puede apreciarse que los Obstetras-ginecólogos tienen como grupo una actitud relativamente conservadora frente a las demás especialidades, excepto los pediatras quienes en un $66.7 \%$ opinan que la legislación debe modificarse.

En relación con la edad, a medida que ésta aumenta, disminuye el porcentaje de los que están de acuerdo con una legislación menos restrictiva. Por otra parte, los encuestados sin hijos, un $62.5 \%$, abogaron por leyes menos restrictivas en contraste con el $100 \%$ de los encuestados con hijas mujeres únicamente. Al cruzar las mismas opiniones sobre legislación con la religión de los encuestados:

\section{CUADRO No. 8}

Se aprecia alguna diferencia entre los que no practican ninguna religión y los católicos o aquellos que practican otras religiones.

La encuesta incluye 4 preguntas que buscaban medir un poco el nivel de conocimiento o la opinión del cuerpo médico sobre la magnitud y jerarquía del aborto frente a otros problemas de salud materna en la ciudad de Bogotá. A estas preguntas los encuestados respondieron colocando el aborto a la cabeza de los problemas de salud materna y también en el primer lugar entre las causas de mortalidad en este grupo de población.

$75 \%$ opina que en la ciudad ocurren entre 10 y 30 abortos por cada 100 embarazos.

\section{CUADRO No. 7}

\section{OPINION FAVORABLE A UNA LEGISLACION MENOS RESTRICTIVA POR ESPECIALIDAD MEDICA}

Salud Pública

Medicina Interna

Psiquiatría 


\section{CUADRO No. 8 \\ OPINION FAVORA]3LE A UNA LEGISLACION RESTRICTIVA POR PRACTICA RELIGIOSA}

No practicantes

\section{CUADRO No. 9}

\section{ACTITUDES}

En ningún caso y bajo ninguna condición permitirse la práctica del aborto

El aborto podría permitirse cuando la continuación del embarazo constituye grave peligro para la vida de la madre.

El aborto podría permitirse cuando el producto de la concepción tiene grandes posibilidades de nacer con problemas físicos 0 mentales graves.

\section{CUADRO No. 10}

\section{ACTITUDES}

El aborto podría permitirse en caso de violación

El aborto podría permitirse cuando la continuación del embarazo implica problemas psiquiátricos, económicos o sociales para la madre.

El aborto podría permitirse con otras condiciones diferentes a las ya mencionadas. 
En lo que respecta a las actitudes de los médicos encuestados frente al problema del aborto:

\section{CUADRO No. 9 CUADRO No. 10}

El $85 \%$ opinó que el aborto debe permitirse cuando la continuación del embarazo constituye grave peligro para la vida de la madre, $79 \%$ cuando el producto de la concepción tiene grandes posibilidades de nacer con problemas físicos o mentales graves, y el $59 \%$ en caso de violación o cuando existan problemas psiquiátricos, económicos o sociales inherentes a la continuación del embarazo.

La actuación personal de los encuestados, si la práctica del aborto fuera permitida bajo alguna de las circunstancias antes mencionadas, se puede apreciar en el siguiente cuadro. la especialidad médica directamente relacionada con el sistema reproductivo, la par. ticipación personal aumenta sustancialmen te.

Finalmente y al cruzar la actuación per. sonal con la religión del encuestado, encon. tramos que las tasas de participación directa disminuyen, persistiendo sin embargo un $21.8 \%$ de médicos que habiéndose declarado católicos practicantes realizarían el aborto ellos mismos, y un $60.2^{\circ}$ o que preferiría remitirlo a otro médico.

\section{RESUMEN Y CONCLUSIONES}

Se presentan los resultados de la encuesta enviada a 2.557 médicos de Bogotá con el fin de conocer sus actitudes y opiniones relacionadas con el aborto.

\section{CUADRO No. 11}

ACTUACION PERSONAL

\section{SI LA PRACTICA DEL ABORTO FUERA PERMITIDA}

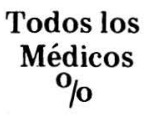

3.4
Obstetras Ginecólogos ç $\%$

No lo practicaría ni lo remitiría bajo ninguna circunstancia

Preferiría remitirlo a otro médico

Lo realizaría personalmente en circunstancias especiales

Lo realizaría personalmente en.todas las circunstancias

Más de una respuesta

Mientras el $24.4 \%$ afirma que realizaría personalmente la interrupción del embarazo, el $59.9 \%$ preferiría remitirlo a otro médico y el $9.9 \%$ no lo realizaría ni lo referiría bajo ninguna circunstancia.

En el caso específico de los Gineco-obstetras, como es de esperarse por tratarse de
La encuesta fue respondida por $648 \mathrm{me}$ dicos lo cual corresponde al $25.3 \%$ de lo encuestados, y por esta razón no puede ase gurarse que la posición de todos los méd cos de Bogotá sean necesariamente similar Sin embargo este estudio constituye êl pr mer esfuerzo importante para conocer posición de los médicos de Bogotá, con res 
pecto a un tema de gran actualidad.

Con relación a la legislación vigente el $10.7 \%$ opinó que se mantuviera igual o se hiciera más restrictiva. El $89.3 \%$ piensa que debe hacerse menos restrictiva.

Respecto a la actitud de los médicos ante la práctica del aborto se encontró que el $10 \%$ nunca lo practicaría, el $60 \%$ lo remitiría a otros médicos para que lo practicaran y el $25 \%$ sí lo practicaría.

El $91 \%$ considera que el aborto es un problema muy grave de salud que puede prevenirse con programas masivos de educación y servicios de planificación familiar.

En conclusión podemos afirmar que a pesar de los esfuerzos que se han realizado para estudiar problemas asociados con el aborto, son pocas las respuestas a muchos de los interrogantes que tanto los estudiosos como el estado se plantean.

El estudio que ahora se entrega presenta una pauta impol ante sobre el tema ya que se hace el intento de conocer la opinión de uno de los sectores al que corresponde una participación más directa en el estudio, prevención y soluciones al problema. En este sentido la encuesta desarrollada por la Sociedad Colombiana de Obstetricia y Ginecología, constituye un aporte al conocimiento del tema.

\section{SUMMARY AND CONCLUSIONS}

The results of the survey sent to 2.557 doctors in Bogotá, in order to know their attitudes and opinions with respect to abortion, are shown.
648 doctors, that is, $25.3 \%$ of the people inquested, answered the survey, and for this reason it can not be assured that the points of view of all doctors in Bogotá are similar. However, this study is the first significant effort to know the criteria of Bogotá's doctors regarding a subject of current importance.

With respect th the legislation in force, $10.7 \%$ stated that it should remain as it is or that it must be more restrictive.

$89.3 \%$ think that it should be less restrictive.

Regarding the doctors attitude towards practising abortion, it was found that $10 \%$ would never practise it, $60 \%$ would send the patient to other doctors and $25 \%$ would send practise it.

$91 \%$ consider that abortion is a very serious health problem that can be prevented with massive educational programs and family planning assitance.

In conclusion, we can assert that in spite of all efforts made to study the problems related with abortion, few are the answers to the many questions posed by researchers and the State.

The study now handed shows an important guideline on the subject, because it is intended to know the opinion of one of the sectors that have a more direct participation in the study, prevention and solution of the problem. In this sense, the survey carried out by the Sociedad Colombiana de Obstetricia y Ginecología (Obstetrics and Gynecology Colombian Association) represents a contribution to the knowledge of the subject. 